\title{
Wertigkeit des qualitativen Nachweises von Serum-IgG-Antikörpern gegen Campylobacter pylori als Indikator der Magenbesiedlung mit C. pylori
}

\author{
Significance of IgG-Antibody Determination to Campylobacter pylori as Indicator of \\ C. pylori colonisation of the Stomach \\ G. von Recklinghausen', R. B. Zotz', R. Ansorg' \\ 'Institut für Medizinische Mikrobiologie \\ ${ }^{2}$ Abteilung für Gastroenterologie des Zentrums für Innere Medizin, Universität (GHS) Essen
}

\begin{abstract}
Zusammenfassung:
Mit einer ELISA-Technik wurden IgG-Antikörper gegen Campylobacter pylori bei 81 magen-endoskopierten Patienten bestimmt. Als Antigen diente der saure Glycin-Extrakt eines C. pylori-Referenzstammes. Die Reaktivität einer $1: 500$-Verdünnung des Patientenserums im ELISA wurde mit dem Nachweis von C. pylori durch Kultur, Mikroskopie und Biopsie-Urease-Test aus zwei Magenbiopsien des Patienten verglichen. Bei einem Extinktions-Grenzwert von 0,500 waren 49 der 53 Patienten mit C. pylori-Besiedlung positiv im ELISA. 22 der 28 Patienten ohne C. pylori-Nachweis in der Biopsie waren auch serologisch negativ. Für das gewählte Testsystem zeigen sich folgende Qualitätsmerkmale: diagnostische Sensitivität $93 \%$, diagnostische Spezifität $79 \%$, positiver Vorhersagewert $89 \%$, negativer Vorhersagewert $85 \%$ und diagnostische Effizienz $88 \%$. Die qualitative Bestimmung der IgG-Antikörper gegen C. pylori ist ein praktikabler Suchtest für die C. pylori-Besiedlung des Magens.
\end{abstract}

Schlüsselwörter:

Campylobacter pylori - IgG-ELISA - Magenbesiedlung

Summary:

IgG Antibody to Campylobacter pylori was measured in the sera of 81 patients undergoing upper gastrointestinal endoscopy. An ELISA technique with acid glycine extracted antigen was used. The reactivity of a $1: 500$ serum dilution in the ELISA was compared to $C$. pylori colonization of the stomach as indicated by culture of C. pylori, Gram stain, and urease testing from stomach biopsies. Using a cutoff of OD 0,500, 49 out of the 53 patients with $C$. pylori colonization were positive in the ELISA. Of 28 patients without C. pylori detection, 22 showed negative serological reactions. Performance criteria were as follows: sensitivity $93 \%$, specificity $79 \%$, positive predictive value $89 \%$, negative predictive value $85 \%$, and efficiency $88 \%$. Measurement of specific lgG antibody to $C$. pylori could be a valuable screening test for the $C$. pylori colonization of the stomach.

Keywords:

Campylobacter pylori - IgG-ELISA - stomach colonization

\section{Einleitung}

Campylobacter pylori (CP) ist ein mikroaerophiles gramnegatives gebogenes Stäbchenbakterium, das eng mit der Typ-B-Gastritis und dem peptischen Ulkus assoziiert ist $(2,7,9)$. Die Diagnose einer CP-Besiedlung des Magens erfordert die Gewinnung von Magenbiopsien, um den Keim kulturell, morphologisch und/oder biochemisch anhand seiner Urease-Aktivität nachzuweisen. Diese invasive Diagnostik ist belastend für den Patienten, zeitaufwendig und kostenträchtig. Daher ist ein einfacher Indikator der C. pylori-Besiedlung erforderlich.

Zur Zeit bieten sich zwei nicht-invasive Verfahren als Suchtests an: der Nachweis von $C_{13}$ in der Atemluft nach einem Testmahl mit markiertem Harnstoff $(10,22)$ und der Nachweis spezifischer Serum-Antikörper $(3,8,13,19)$. Der Harnstoff-Atemtest ist einfach in der Durchführung, eine relativ aufwendige Ausrüstung und Akzeptanzprobleme beim Patienten behindern jedoch seine Verbreitung. Die serologische Untersuchung ist daher für die routinemäßige Laboratoriumsdiagnostik praktikabler.
CP löst eine systemische und lokale Immunantwort aus $(14,26)$. Komplement-Bindungs-Reaktion, Direkt-Agglutination, passive Hämagglutination, Enzym-Immuno-Assay und Immunoblot-Techniken wurden zum Nachweis der spezifischen Immunreaktion beschrieben $(8,13,14,16$, 24, 25). Bei der klassenspezifischen Untersuchung von Serum-Antikörpern wurde bei vorangehenden Studien eine gute Übereinstimmung der spezifischen IgG- und IgA-Antikörper-Titer mit der CP-Besiedelung und der Antrum-Gastritis nachgewiesen. Akutphase-Antikörper der IgM-Klasse korrelierten allerdings nicht mit dem Keimnachweis $(7,13)$.

Ziel der vorliegenden Untersuchung ist, die qualitative Bestimmung von Serum-lgG-Antikörpern gegen CP als Suchtest für eine CP-Besiedlung des Magens zu evaluieren.

\section{Material und Methoden}

\section{Patienten}

81 Patienten der endoskopischen Abteilung der Medizinischen Klinik der Universität Essen wurden zwischen April 
und Dezember 1988 untersucht. Zum Nachweis der CPBesiedelung wurden je zwei Magenbiopsien überwiegend aus dem Antrum-Bereich entnommen. Parallel wurde Serum gewonnen und bis zur Untersuchung bei $-20^{3} \mathrm{C}$ gelagert.

\section{Nachweis der CP-Besiedelung des Magens}

Ein Biopsat wurde sofort nach der Entnahme in ein Urease-Testmedium (1) gegeben und nach $20 \mathrm{~min}, 3$ und $24 \mathrm{~h}$ auf Farburnschlag abgelesen. Das zweite Biopsat wurde in Fildes-Medium innerhalb von $48 \mathrm{~h}$ zum mikrobiologischen Labor transportiert und folgendermaßen verarbeitet (21): Das Gewebestück wurde mäanderförmig auf Schafblut-Agar, TTC-Agar (20) und Serum-AktivkohleAgar (6) ausgestrichen. Ein Objektträgerausstrich wurde nach Gram gefärbt und auf gramnegative gebogene Stäbchen durchmustert. Die Medien wurden nach mikroaerophiler Inkubation $\left(\mathrm{CO}_{2} 8-10\right.$ vol\%, $\mathrm{O}_{2} 5-7$ vol\%, Rest $\mathrm{N}_{2}$, Anaerocult C, Merck, Darmstadt) bei $37^{\circ} \mathrm{C}$ im Zeitraum zwischen drei und zwölf Tagen mehrfach auf verdächtige Kolonien untersucht und diese auf Urease-, Katalase- und Oxidase-Aktivität geprüft. Ein Biopsat wurde als CP-haltig bezeichnet, wenn die Kultur, die Mikroskopie und/oder der Biopsie-Urease-Test positiv ausfielen.

\section{Enzyme-linked immunosorbent Assay (ELISA)}

IgG-Antikörper gegen CP im Serum wurden wie folgt bestimmt $(15,18,23)$ : C. pylori ATCC 43504 wurde 48-72 h auf Schafblut-Agar unter mikroaerophilen Bedingungen gezüchtet, mit $\mathrm{H}_{2} \mathrm{O}$ geerntet und zweimal gewaschen. Das Bakterienmaterial von drei $8,5 \mathrm{~cm}$-Agarplatten mit dichtem Wachstum wurde in $12 \mathrm{ml}$ Glycin-HCl-Puffer 0,2 $\mathrm{mol} / \mathrm{l}(\mathrm{pH} \mathrm{2,2)}$ resuspendiert und $15 \mathrm{~min}$ bei Raumtemperatur (RT) gerührt. Nach Zentrifugation für $20 \mathrm{~min}$ bei $10000 \mathrm{~g}$ wurde der Überstand mit $\mathrm{NaOH}$ auf pH 9,0 alkalisiert. Der Proteingehalt der Lösung (12) lag bei $5 \mathrm{mg} / \mathrm{l}$. Mikrotiterplatten (ImmunoPlates Maxisorp F 96, NUNC, Roskilde, DK) wurden mit je $200 \mu$ l Antigenextrakt pro Vertiefung beschickt, zwei Std. bei $37^{\circ} \mathrm{C}$ und über Nacht bei $4^{\circ} \mathrm{C}$ inkubiert. Antigen-beschichtete Platten wurden bei $4^{\circ} \mathrm{C}$ gelagert und innerhalb einer Woche verbraucht.

Vor Gebrauch wurden die Platten dreimal in 0,066 mol// Phosphatpuffer (pH 7,0) plus 0,1\% Tween 20 (PBS-T) gewaschen.Die Patientenseren wurden in PBS-T mit $1 \%$ Rinderserum-Albumin (Merck) 1 : 500 verdünnt. Je $200 \mu \mathrm{l}$ der Serumverdünnungen wurden in die Näpfchen der an- tigenbeschichteten Platten pipettiert und $90 \mathrm{~min}$ bei $37^{\circ} \mathrm{C}$ inkubiert. Nach Waschen mit PBS-T wurde mit je $200 \mu l$ Peroxidase-konjugiertem affinitätsgereinigtem gammaspezifischem Anti-Human-Serum von der Ziege (Kierkegaard \& Perry, Asbach) $90 \mathrm{~min}$ bei $37^{\circ} \mathrm{C}$ inkubiert. Die optimale Konjugat-Konzentration lag bei 1-10 $\mu \mathrm{g} / \mathrm{l}$ Antikörperprotein. Nach erneutem Waschen wurde mit je $200 \mu \mathrm{l}$ ABTS- $\mathrm{H}_{2} \mathrm{O}_{2}$-Substrat (Kierkegaard \& Perry) $30 \mathrm{~min}$ bei RT inkubiert und die Extinktion bei $492 \mathrm{~nm}$ auf einem Mikrotiterplatten-Photometer (MR 700, Dynatech, Denkendorf) gemessen.

\section{Kreuzreaktivität des Antigens}

Zur Prüfung der Reaktivität des Test-Antigens mit kreuzreagierenden Antikörpern wurde eine 1 : 500-Verdünnung eines positiven Patientenserums mit dem homologen C. pylori-Stamm und je einem Referenzstamm von C. jejuni, E. coli, S. faecalis, S. aureus und C. albicans in einer Keimzahl von $10^{7} / \mathrm{ml} 30 \mathrm{~min}$ bei RT absorbiert. C. pylori reduzierte die Reaktivität des Serums mit dem Testantigen um $75 \%$. Die anderen geprüften Spezies reduzierten die Reaktivität wie folgt: C. jejuni $12 \%$, E. coli $16 \%$, S. faecalis $12 \%, \mathrm{~S}$. aureus $6 \%, \mathrm{C}$. albicans $5 \%$.

\section{Qualitätskontrolle und Statistik}

Die Testseren wurden doppelt angesetzt, in jedem Lauf wurde ein reaktives, ein nicht reaktives und zwei grenzwertige Patientenseren mitgeführt. Der Signifikanztest basiert auf der t-Verteilung. Bei der Berechnung der Standardabweichung wurden drei Ausreißerwerte vernachlässigt. Die Qualitätskriterien wurden nach Galen berechnet (5).

\section{Ergebnisse}

Bei 81 Patienten wurde das Vorhandensein von SerumIgG-Antikörpern gegen Campylobacter pylori (CP) mit dem Nachweis von CP im Magenbiopsat verglichen. Bei $53(65 \%)$ Patienten wurde eine Besiedlung mit CP nachgewiesen (CP-positive Patienten), wobei 30 in der Kultur, 32 mikroskopisch im Ausstrichpräparat und 45 im Biopsie-Urease-Test positiv ausfielen. 28 (35\%) Patienten waren CP-negativ.

Der Extinktionswert des IgG-Antikörper-ELISA-Tests wurde dem Ergebnis der bioptischen Untersuchung zugeordnet (Abb. 1). Es zeigt sich eine überwiegende Zuordnung der hohen Extinktionswerte zu den CP-positiven $\mathrm{Pa}$ -

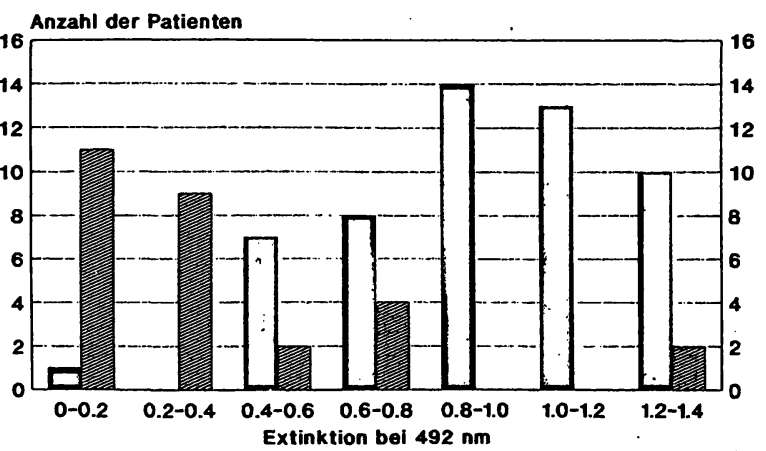

c. pylori positiv c. pylori negativ

Abb. 1: Zusammenhang zwischen C. pylori IgG-AntikörperELISA (Extinktionswerte) und bioptischem C. pylori-Nachweis (Kultur, Mikroskopie, Urease-Test).

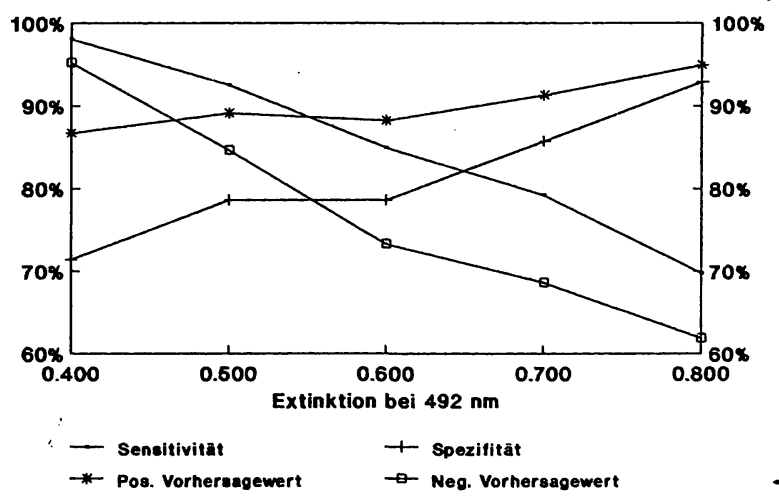

Abb. 2: Qualitätsparameter des ELISA zum Nachweis von IgGAntikörpern gegen Campylobacter pylori bei unterschiedlichen Extinktionsgrenzwerten. 
tienten. Der Mittelwert der Extinktionswerte von $52 \mathrm{CP}$. positiven Patienten liegt mit 0,941 (Standardabweichung 0,261 ) signifikant über dem Wert von 0,294 (Standardabweichung 0,200 ) bei 26 CP-negativen Patienten $(p<$ 0,001).

Zur Ermittlung des Extinktionswertes, der eine optimale Trennung der positiven und der negativen Gruppe bietet, wurden die Sensitivität, die Spezifität, der positive und der negative Vorhersagewert sowie die Effizienz des ELISA bei den Grenzwerten $0,400,0,500,0,600,0,700$ und 0,800 berechnet (Abb. 2). Der Grenzwert 0,400 bietet eine hohe diagnostische Sensitivität von $98 \%$ und einen hohen negativen Vorhersagewert von $96 \%$, jedoch eine mäßige diagnostische Spezifität von $72 \%$. Bei einem Grenzwert von 0,800 sinkt die diagnostische Sensitivität auf $70 \%$, während die diagnostische Spezifität $93 \%$ erreicht. Um eine optimale Effizienz des ELISA zu erreichen, wurde der Grenzwert auf 0,500 gesetzt. Damit werden die folgenden Qualitätskriterien erreicht: diagnostische Sensitivität $93 \%$, diagnostische Spezifität $79 \%$, positiver Vorhersagewert $89 \%$, negativer Vorhersagewert $85 \%$ und diagnostische Effizienz $88 \%$.

Zur Befunderstellung wurden drei Extinktionsbereiche festgelegt: der Bereich zwischen 0 und 0,400 wurde als negativ, der Bereich zwischen 0,400 und 0,800 als grenzwertig und der Bereich über 0,800 als positiv gewertet.

39 Patienten zeigten Extinktionswerte über 0,800. Bei 37 dieser Patienten wurde CP nachgewiesen. Die beiden CP. negativen Patienten in dieser Gruppe hatten im einen Fall eine chronisch atrophische Gastritis mit intestinaler $\mathrm{Me}$ taplasie, im anderen Fall eine aktive Oberflächengastritis. In der Gruppe mit Werten zwischen 0,400 und 0,800 waren $15 \mathrm{CP}$-positive und $7 \mathrm{CP}$-negative Patienten. Bei 8 der 15 CP-positiven Patienten gab es Hinweise auf eine geringe Besiedelungsdichte in den Biopsaten, z. B. niedrige Keimzahlen bei der mikroskopischen Beurteilung oder langsame Harnstoff-Spaltung im Biopsie-Urease-Test. 20 der 21 Patienten mit ELISA-Werten unter 0,400 waren CPnegativ. Der einzige Patient mit einem falsch negativen ELISA-Ergebnis zeigte nur einige gramnegative gebogene Stäbchen im Biopsie-Ausstrich; Kultur, BiopsieUrease-Test und histologische Gastritis-Diagnostik waren negativ.

\section{Diskussion}

Der Nachweis von spezifischen IgG-Antikörpern im $\mathrm{Pa}$ tientenserum wird als ein zuverlässiger Indikator für eine Besiedelung des Magens mit Campylobacter pylori (CP) und für eine Antrumgastritis beschrieben $(8,19)$. Auch im vorliegenden Kollektiv von Patienten einer endoskopischen Abteilung korreliert der Nachweis von IgG-Antikörpern hoch mit der CP-Besiedelung.

Der qualitative IgG-ELISA ermöglicht eine signifikante Trennung der CP-negativen von den CP-positiven Patienten. Die diagnostische Sensitivität des Tests erreicht über $90 \%$, die diagnostische Spezifität knapp $80 \%$. Beim positiven Vorhersagewert von $89 \%$ ist jedoch die hohe Prävalenz der CP-Besiedelung in unserem Kollektiv zu berücksichtigen. Bei einer asymptomatischen Population wurde eine Prävalenz von etwa $20 \%$ gefunden (11). Bezogen auf diese Prävalenz würde sich der positive Vorhersagewert des Tests auf $70 \%$ reduzieren.

Die Aufarbeitung des Antigens folgt im wesentlichen den Angaben von Newell et al. (18). Die saure Extraktion der äußeren Membranproteine hat sich gegenüber Ganzzellpräparationen oder ultraschallbehandelten Zellen als überlegen erwiesen, da eine mögliche Kreuzreaktivität mit Antikörpern gegen flagelläre Proteine von $C$. jejuni im Patientenserum reduziert wird (17). Bei der vorliegenden Methode wurde die Antigen-Präparation durch Verzicht auf eine Dialyse verkürzt. Offenbar ist bei Verwendung von frischen Antigen-Präparationen ohne Einfrieren oder Lyophilisation die Antikörper-Bindung trotzdem ausreichend und reproduzierbar.

Die Reproduzierbarkeit der Extinktionswerte bei konstanten Reaktionsbedingungen hängt wesentlich von der gleichbleibenden Konzentration des Konjugats ab. Beim gleichen Verdünnungsansatz und Portionierung des Konjugats sind die Werte gut reproduzierbar. Bei einem Neuansatz der Konjugat-Verdünnung sollte jedoch eine Eichung mit Patientenseren aus verschiedenen Extinktionsbereichen durchgeführt werden. Die Herstellung des Antigen-Extrakts aus dem abgeernteten Zellmaterial dauert rund drei Stunden, das gesamte Protokoll inclusive Antigen-Extraktion kann in zwei Tagen durchgeführt werden.

Obwohl der Test für die überwiegende Zahl der Proben eine richtige und eindeutige Zuordnung ermöglicht, bleibt ein gewisser Prozentsatz an grenzwertigen Ergebnissen. Ob diese grenzwertige Reaktivität durch interferierende Antikörper oder durch Abfall der spezifischen Antikörper post infectionem bedingt ist, läßt sich zur Zeit nicht entscheiden, da kontrollierte Verlaufsstudien bisher fehlen. Die Kreuzreaktivität unseres Antigens mag in einigen Fällen zu grenzwertigen Ergebnissen führen, da die Testung mit einigen häufigen Erregern inclusive $C$. jejuni zum Teil leichte Reaktivitätseinbußen nach Absorption ergab.

Für die Befunderstellung empfiehlt sich die Festlegung eines Grenzwertbereiches. Für die vorliegende Testanordnung wurde der Bereich zwischen 0,400 und 0,800 festgelegt. Das hat zur Folge, daß $26 \%$ unserer Proben als grenzwertig befunden wurden. Eine Einengung des grenzwertigen Bereichs würde diese Probengruppe verkleinern, allerdings mit dem Risiko vermehrt falsch positiver bzw. falsch negativer Zuordnungen. Der Anteil der grenzwertigen Proben läßt sich möglicherweise durch die Verwendung der gereinigten CP-Urease als Antigen reduzieren (4).

Die vorliegenden Daten zeigen, daß die qualitative serologische Bestimmung von IgG-Antikörpern gegen $C P$ ein praktikabler Indikator für eine $\mathrm{CP}$-Besiedelung des $\mathrm{Ma}$ gens ist. Die serologische Untersuchung ist eine Ergänzung der invasiven Magendiagnostik und kann den invasiven CP-Nachweis in den Fällen ersetzen, in denen die Gewinnung einer Magenendoskopie kontraindiziert oder zu aufwendig wäre.

Der Ėrsatz der gastroskopischen und radiologischen Magendiagnostik durch die spezifische CP-Serologie bei unkomplizierten klinischen Bildern und zur Ausschlußdiagnostik der Gastritis wird diskutiert (9). Patienten mit Beschwerden und gleichzeitig positivem Serotest können gezielt behandelt werden, um die CP-Besiedelung des Magens zu vermindern oder zu beenden. Nur Therapieversager oder Patienten mit Karzinomverdacht oder Anämie müssen sich dann noch einer weitergehenden Diagnostik unterziehen.

Ob der hier vorgestellte ELISA auch zur Verlaufskontrolle der CP-assoziierten Krankheitsbilder; eventuell nach gezielter antibiotischer Behandlung geeignet ist, müssen weitere Untersuchungen klären.

Lab.med. 13: 423 (1989) 
Schriftum:

1. ARVIND, A. S., COOK, R. S., TABAQCHALI, S., FARTHINO, M. J. G.: Onquminute endoscopy room test lor Campylobacter pylori. Loncet I, 704 (1988).

2. BLASER. M. J.: Gestric Cempylobecter-flke organiams, gastrtis, and peptic ulcer disaace. Gastroenterology 93, 371-383 (1987).

3. BOOTH, L.. HOLOSTOCK, G. MACBAIDE, H. HAWTIN, P. GIBSON, J. R., IAELAND, A. BAMFORTH, J. DUBOULAY, C. E., LLOYD, A. S. PEARSON. A. D.: Cllnical importance of Campylobecter pytoridis and associated serum ige and IgA antibody responses in pa. tients undergoing upper oastrointestinal endoscopy. J. Cin. Pathol. 39, 215-218 (1986) 4. DENT, J. C., MENULTY, C. A. M. UFF, J. S., GEAR. M. W. L. WILKINSON, S. P.: Campylobacter pytori urease: a now serological tost. Lancet I, 1002 (1980).

5. GALEN, R. S. Use of predictive value theory in clinical immunology. In: Manual of Clinical Laboratory Immunology. Eds. ROSE, N. R., FRIEDMAN, H., FAHEY, J. L. Amorican Socioty for Microbiology, Washington D. C., $966-970$ (1986).

6. GLUPCZYNSKI, Y, LABBE, M. THIBAUMONT, F: Comparative evaluation of a now seloctive culture medium for Improved isolation of campylobacter pylori from gastric biopsy spocimens. Abstr. Workshop gastroduodenal pathology and Campylobacter pylorl, Bordeaux 1988

7. GOODWIN, C. S., ARMSTRONG. J. A., MARSHALL, B. J: Campylobacter pyloridis, gastritis, and peptic ulceration. J. Clin. Pathol. 39, 353-365 (1986).

8. GOODWIN, C. S., BLINCOW. E., PETERSON, G., SANDERSON, C., CHENG, W., MARSHALL, B., WARREN, J. R., McCULLUCH, R.: Enzymelinked immunosorbent assay for Campylobacter pyloridis: Correlation with presence of $C$. pyloridis in the gastric mucosa. J. Infect. Dis. 155, 488-494 (1987).

9. GRAHAM, D. Y: Campylobacter pylorl and peptic ulcer disease. Gastroenterology 96. 615-625 (1989).

10. GRAHAM, D. Y., KLEIN, P. D., EVANS, D. J., EVANS, D. G., ALPERT, L. C., OPEKUN, A. R., BUTTON, T. W.: Campylobacter pylori detected noninvasively by the 13C-urea breath test. Lancet I. 1174-1177.

11. GRAHAM, D. Y., KLEIN, P. D., OPEKUN, A. R., ALPERT, L. C., KLISH, W. J., EVANS, C. J., MICHALETZ, P. A., YOSHIMURA, H. H., ADAM, E., BOUTTON, T. W.: Epidemiology of Campylobacter pyloridis infection. Gastroenterology 92, 1411 (1987).

12. HARTREE, E. F.: Determination of protein: A modification of the Lowry method that gives a linear photometric response. Anal. Biochem. 48, 422-427 (1972).

13. HIRSCHL, A. M.: Frequency of occurrence of Campylobacter pylori and analysis of the systemic and local immune response. Zbl. Bakt. Hyg. A 266, 526-542 (1987).

14. JONES, D. M., LESSELLS, A. M., ELDRIDGE, J.: Campylobacter like organisms in the gastric mucosa: culture, histological and serological studies. J. Clin. Pathol. 37, 1002-1006 (1984).

15. KRAUS, C., FISCHER, S., ANSORG, R., HÜTTEMANN, U.: Pneumococcal antibodies (IgG, IgM) in patients with chronic obstructive lung disease 3 years after pneumococcal vaccination. Med. Microbiol. Immunol. 174, 51-58 (1985).

16. MARSHALL, B. J., McGECHIE, D. B., FRANCIS, G. J., UTLEY, P. J.: Pyloric Campylobacter serology. Lancet I, 281 (1984).

17. NEWELL, D. G.: Identification of the outer membrane proteins of Campylobacter pyloridis and antigenic cross-reactivity between C. pyloridis and C. jejuni. J. Gen. Microbiol. 133, $163-170$ (1987).

18. NEWELI, D. G., MacBRIDE, H., PEARSON, A. D.: The identification of outer membrane proteins and flagella of Campylobacter jejuni. J. Gen. Microbiol. 130, 1201-1208 (1984).
19. NEWELL, D. O., JOHNSTON. B. J., AL, M. H., REED, P. I.: An enzyme-linked immuno sorbent assey for the serodlagnosis of Cempylobacter pyorl ascoliated gastritis. Scand. J. Gastroenterol. 23/suppl 142, 53-57 (1988).

20. OUEIROZ, D. M. M., MENDES, E. N., ROCHA, G. A.: Indicator medium for isolation of Campylobacter pyior. J. CHn. Microbiol. 25, 2378-2379 (1987).

21. v. RECKLINGHAUSEN, G., KEMLER, U., BREUER, N., ANSORG, R. Arsesesment of

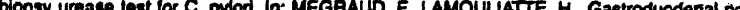
thology and Campylobacter pylort. Excerpta Medica Int Congress Sertes. Ansterdam. $61-63$ (1989).

22. SUSANTO, F. HUMFELO, 8. AEINAUER. H.: Nichtinvasiver Nachwols von Camoyobacter pylor in Patienten mil Antrum Gastritis mittels lsotop-ratio Masisenspeltrometrie. Lab. Mod. 13, 187- 188 (1989)

23. VOLLER, A., BIOWELL, D. E., BAFTLET, A.: Enzyme immunoassays in dlagnostic modicine. Bull. World. Health. Org. 53, 55-65 (1976).

24. v. WULFFEN, H., GROTE, H. J., GATERMANIN, S., LONING, T., BERGER, B., BUHL C.: Immunoblot analysis of immune response to Campylobacter pylori and its clinical assoclations. J. Clin. Pathol. 41, 653-659 (1988).

25. v. WULFFEN, H. HEESEMANN, J, BOTZOW, G. H. LONING, T. LAUFS, R. Detection of Campylobacter pyloridis in patients with antrum gastritis and peptic ulcers by culture. complement fixation test, and immunoblot. J. Clin. Microbiol. 24, 716-720 (1986).

26. WYATT, J. I., RATHBONE, B. J., HEATLEY, R. V.: Local immune response to gastric Campylobacter in non-ulcer dyspepsia. J. Clin. Pathol. 39, 863-870 (1986).

Wir danken A. Blobner, U. Homann und A. Spies für technische Assistenz.

Anschrift für die Verfasser:

Dr. G. von Recklinghausen

Institut für Medizinische Mikrobiologie

Universität (GHS) Essen

Hufelandstraße 55

4300 Essen 1 

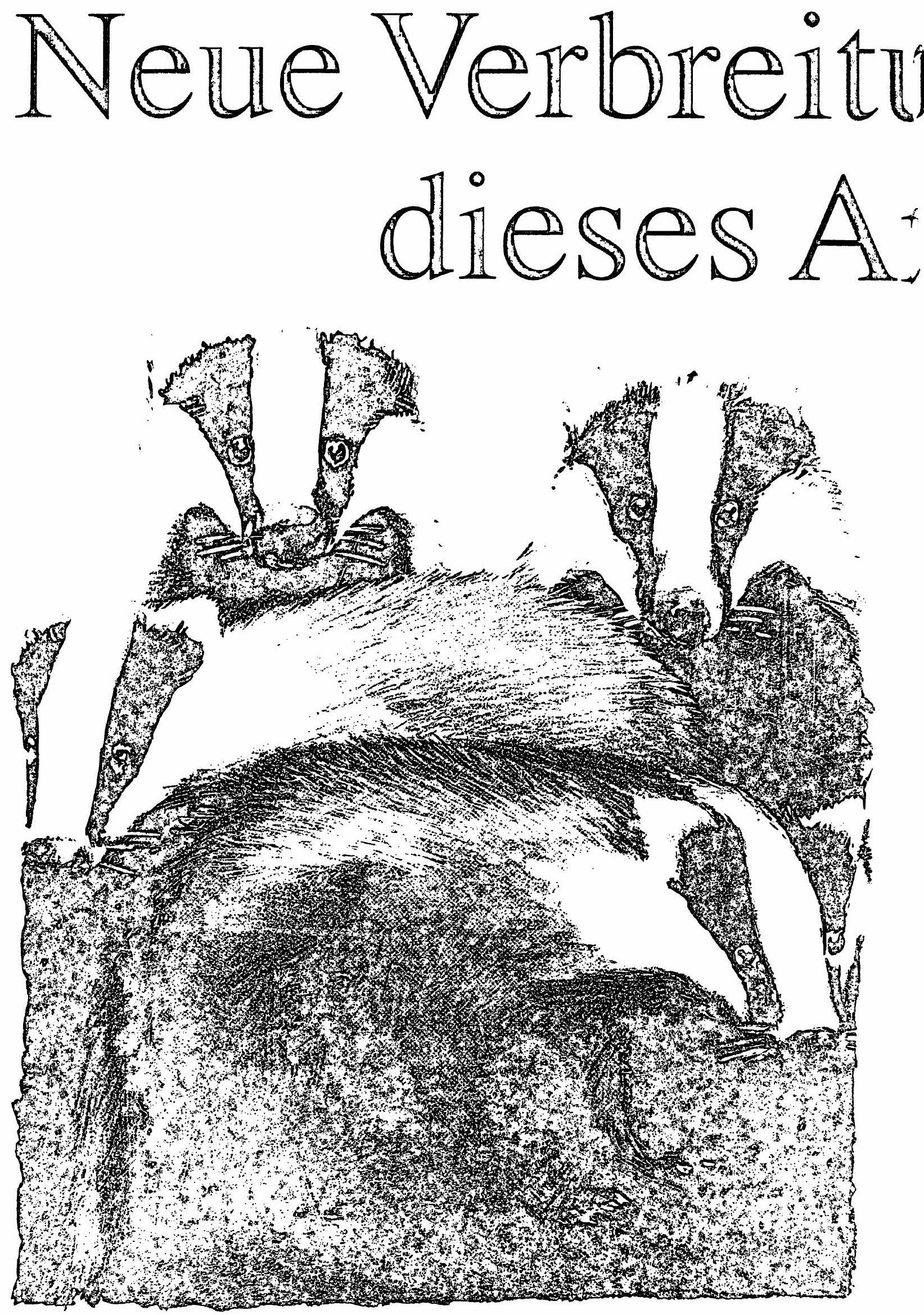


\section{Weder Schweiss noch Tränen}

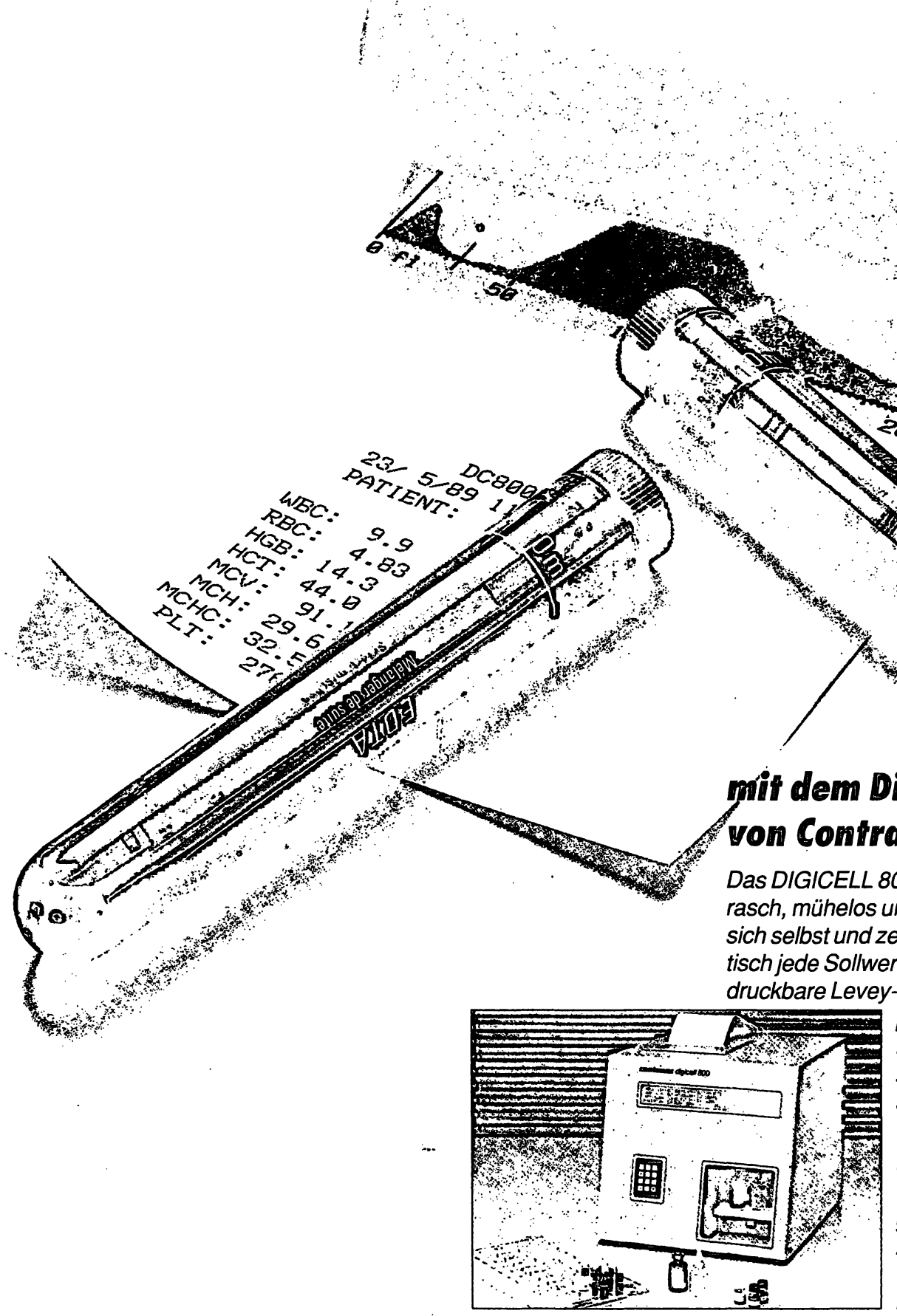

es ermittelt acht Parameter und erstellt zusätzlich RBC/PLT-Histogramme. Der eingebaute Drucker und die EDV-Schnittstelle verhindern zuverlässig Protokollfehler.

DIGICELL 800 bedeutet Bestimmungen mit wenig Blut und ohne Schweiss und Tränen.

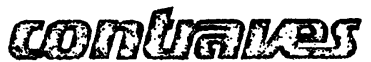

Contraves AG, Schaffhauserstrasse 580, Postfach, CH-8052 Zürich, Telefori 01/8294111, Telex 823402 , Fax $01 / 8109105$ 